\title{
9 \\ Water Quality Management using a Fuzzy Inference System
}

\author{
Kumaraswamy Ponnambalam and Seyed Jamshid Mousavi
}

A fuzzy inference system (FIS) is presented for the optimal operation of a reservoir system with the objective of maintaining water quality in a creek. An optimization model determines optimal reservoir releases for given inflows, initial storage volume and required values for flow augmentation. These optimal releases are then used as inputs to derive optimal operating policies that are easy to use. FIS uses samples of optimal releases and corresponding system variables, namely inflows and storage volumes, to establish the general operating policies. Results compare the superior performance of the proposed algorithm with policies developed using a multiple regression analysis.

\subsection{Introduction}

Flow augmentation is an important objective of reservoirs in urban areas. Controlled reservoir releases are necessary if water must be stored during high flows and released during low flows to keep pollutant concentration below the required limit in streams. Typical situations may require more than one reservoir. In addition, for simplicity of operations and for fast actions, easy to use operating policies are necessary in the hands of operators. In general, optimal releases are derived using complex optimization algorithms especially in the case of uncertainty in inflows. Such algorithms are not easily usable in

Ponnambalam, K. and S.J. Mousavi. 2001. "Water Quality Management using a Fuzzy Inference System." Journal of Water Management Modeling R207-09. doi: 10.14796/JWMM.R207-09.

(c) CHI 2001 www.chijournal.org ISSN: 2292-6062 (Formerly in Models and applications to Urban Water Systems. ISBN: 0-9683681-4-X) 
real-time. Therefore, the objective of this work is to derive effective and relatively easy-to-operate policies that can be distributed to reservoir operators, but the policies themselves would be derived using state-of-the-art optimization algorithms, for example, as in Fletcher and Ponnambalam (1998).

\subsection{Policy Determination}

The optimization model commonly used has an objective function and a set of constraints and is generally deterministic. In the problem here we are dealing with inflows that are stochastic. When one or more variables are uncertain or stochastic, we may need to consider explicitly the uncertainty in the optimization method. Implicit stochastic optimization is a method where the original deterministic optimization is solved repeatedly for each sample of the stochastic variables called the scenario. Although optimal solutions are available for each of these it is not clear how to combine these results so that a simple release policy can be determined. One of the simplest methods is to use multiple linear regression to relate the decision variable to the observed input variables. For highly nonlinear problems and for problems with high uncertainty, this method may not yield satisfactory results. In this chapter we propose an alternative method called the fuzzy inference system (FIS), which is based on "if-then" rules of fuzzy variables, the Fuzzy Rule-Based (FRB) model. A major advantage of these rules is that they are simple to implement and are supposedly able to mimic human thought processes. Because of the high degree of nonlinearity involved and the potentially large number of parameters that need to be determined, the method requires a robust parameter estimation method (Jang, 1993).

\subsection{Proposed Methodology}

We will first describe the objective of optimization. If we assume that the inflows and the initial storage volume are known then releases can be determined such that, for example, they minimize the sum of squared deviations of releases from target releases. The target releases may be determined to reduce the pollutant concentration over a time horizon to below the required limit. The main constraints are physical bounds on storage volumes and releases, and also on the volume conservation equation. However, the inflow for the given horizon may be known only statistically, that is, each scenario has a given probability of occurrence. Therefore, it is possible to get a set of optimal releases only for the given inflow scenario. Many such scenarios can be 
generated to determine the corresponding optimal releases. Now, the simple question is: which one of these policies should be used in the future? For example, if we had NT number of optimal releases, each corresponding to an inflow scenario, how do we combine these results to get an operating policy? This has been a difficult question that is often answered only heuristically except in some cases, for example dynamic programming (DP) where the results of optimization are the operating policies. However, DP is hard to solve especially in the case of multi-state variable systems and hence is not considered here.

\subsection{Regression Analysis}

Given the various inflow scenarios and the corresponding optimal releases and storage volumes (at the beginning of a given period) a multiple regression analysis can be used in the following manner. In Equation 9.1 below we need to determine the coefficients $a, b, c, d$, and $e$, which can be found by linear leastsquares method.

$$
R_{t}=a I_{t}+b S_{t}+c I_{t}^{2}+d S_{t}^{2}+e
$$

where:

$$
\begin{aligned}
R_{t} & =\text { the release during period } t \\
I_{t} & =\text { the inflow during period } t \\
S_{t} & =\text { and }
\end{aligned}
$$

In Equation 9.1 there are no cross terms between inflow and storage because of the statistical independence of inflow on storage. This simple method provides an easy to use policy for reservoir operators and the results of this method will be compared to the results of the proposed fuzzy inference system described next.

\subsection{Fuzzy Inference System}

The fuzzy inference system is a framework to simulate the behavior of a given system through the knowledge of the experts or past available data of the system. The inference engine of a FIS includes a set of fuzzy "if-then" rules and is also called the fuzzy rule-base (FRB). Fuzzy "if-then" rules have the form 
Here, the value of variable reservoir storage, called the 'premise' variable, influences the release value, called the 'consequent' variable. However, both these variables are understood in a non-crisp manner, that is, we can be somewhat imprecise about what value should be considered high and hence these are called fuzzy "if-then" rules. Also, note that the reservoir storage and release are linguistic variables where "high" is a linguistic label. But in reality, we may have specific values measured for the reservoir storage and we need to give specific instructions as to what the release should be. The fuzzification of crisp data and defuzzification of fuzzy decisions are integral parts of the fuzzy inference system. In an alternative form of FIS, called the Takagi-Sugeno (Sugeno, 1985) type, which we use in this chapter, fuzzy "if-then" rules have the form:

\section{If reservoir storage is high, release $=a *$ reservoir storage $+b$}

where the consequent part is crisp, but has two parameters that need to be estimated. So far we used just one premise variable but these type of rules can be extended to multiple premise variables, for example,

$$
\begin{aligned}
& \text { If reservoir storage is medium and inflow is low, } \\
& \text { release }=a * \text { reservoir storage }+b \text { *inflow }+c
\end{aligned}
$$

Once again, the parameters $a, b$, and $c$ must be estimated using available data or operator experience. We describe next the various steps involved in a TakagiSugeno type FIS.

\subsubsection{Fuzzification}

This is the first step in a FIS and is used to fuzzify input premise variables using fuzzy membership functions. This is shown in Figure 9.1. For example, in the top-left of the figure we see that for a given value of S (storage), the membership degree in the state of the storage premise variable for rule 1 is $\mathrm{w}^{\mathrm{S}}$. Similarly, for the other premise variable I (inflow) we find that the membership degree in the state of the Inflow premise variable for rule 1 is $\mathrm{w}^{\mathrm{I}}$. The top row of two figures in Figure 9.1 with the T-norm operator (described below) and the corresponding consequent part, a linear combination of premise variables in the Takagi-Sugeno FIS form, defines one fuzzy "if-then" rule. It should be noted that each premise variable is represented by a linguistic variable. A linguistic variable is a variable such that its value is a word, for example, "low", "medium", and "large" rather than a crisp number; and a fuzzy number or its membership function represents each word. Therefore, each premise (linguistic) 


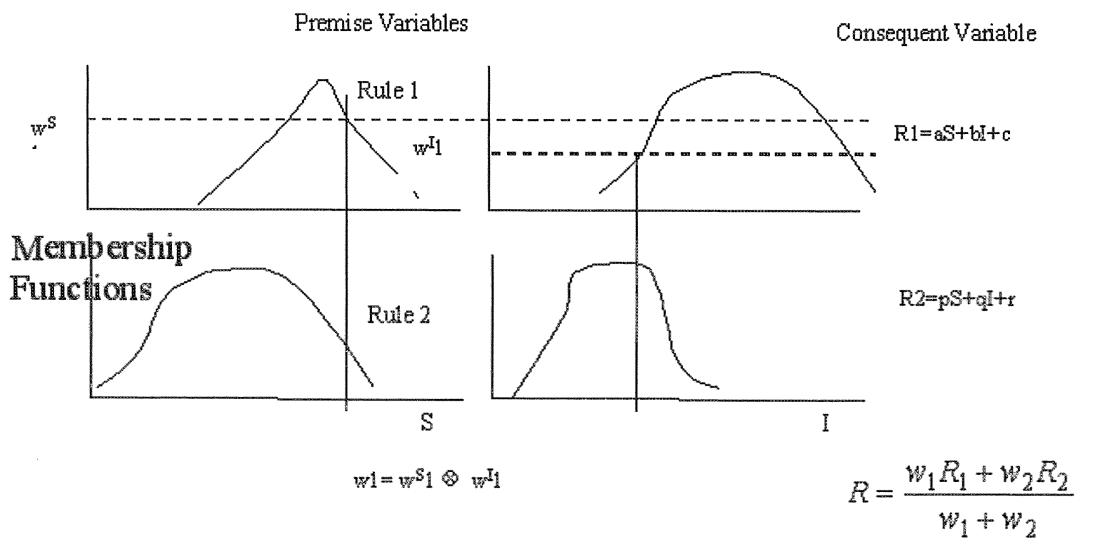

Figure 9.1 Fuzzy interface system (FIS).

variable is divided into some overlapping clusters, which are shown as an example in Figure 9.2. For example, the membership values of storage value $=3$ corresponding to Low, Medium, and High clusters are 0.3, 0.98, and 0.08, respectively. The shape of the membership function here is defined by a parametric function such as a Gaussian function. The selection of number of clusters and their values for each premise variable and the shape tuning of membership functions should be done in an efficient way. We discuss this task under parameter estimation.

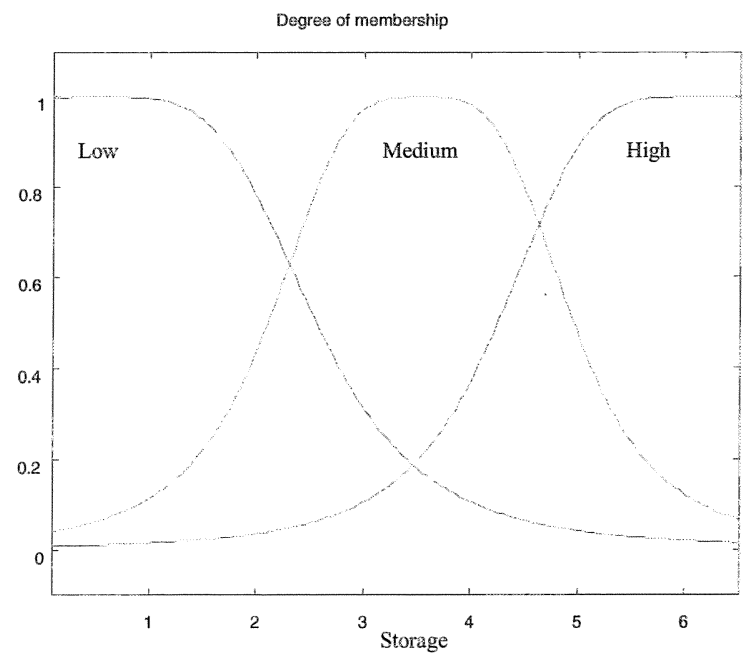

Figure 9.2 Clustering of input variable and its membership functions. 


\subsubsection{Fuzzy Operation}

The objective of this step is to combine the two fuzzy membership values for the two premise variables into one weight (called the firing strength or weight) for this particular decision. The type of required fuzzy operation depends on the type of inference, which is a function of the system's behavior. Two general fuzzy operations are "AND" (intersection) and "OR" (inclusion). There are different fuzzy operators such as "min" or "product" for the "AND" and different operators such as "max" or "algebraic sum" for the "OR" operations. In general, the "AND" operators are called T-norm $(\otimes)$ and "OR" operators are called S-norm or T-conorm $(\oplus)$. Also depending upon the type of application, there are parameterized T-norms and S-norms. These can be found in the literature (Zimmermann, 1984). For example, in Figure 9.1, if we use the "product" T-norm operator, then we get a weight of $w_{1}=w^{S} \times w_{1}^{I}$, for rule 1 . This weight can be used when aggregating the results of the all the "if-then" rules of the given FIS as described next.

\subsubsection{Aggregation}

If we have $i=1 \ldots \mathrm{m}$ "if-then" rules, their corresponding weights $w_{i}$, and decisions $R_{i}$, then we can aggregate them to get the decision $R$ as follows:

$$
R=\frac{\sum_{i=1}^{m} w_{i} R_{i}}{\sum_{i=1}^{m} w_{i}}
$$

Due to the nonlinear membership functions and the nonlinearity of the Tnorm operator in general, this decision is a nonlinear combination of input variables and hence is quite different from regression-based release rules which are usually a linear combination of variables and their powers. This may be the reason why these rules work better for reservoir operations problems, which are nonlinear problems due to the presence of upper and lower bounds on storage even if the reservoir continuity equation is linear (Fletcher and Ponnambalam, 1998). In Takagi-Sugeno type FIS, the aggregated decision $R$ is a crisp value and we do not need the defuzzification step; otherwise a defuzzification method is used to get a crisp value from the aggregated decision. 


\subsubsection{Parameter Estimation}

The type and shape of membership functions, number of clusters, parameters of the consequent part of fuzzy rules and other characteristics of a given FIS should be defined using the knowledge of experts or available input-output data or a combination of both. Defining a FIS using the knowledge of experts as an Initial FIS and then training this FIS through the input-output data is an effective way to obtain the best results. While the above steps define the fuzzy "if-then" rules to model the release decision as a function of input variables storage and inflow, there are many parameters still to be estimated. For example, for a Gaussian membership function we need two parameters, and in this case most data require two to five clusters with each cluster requiring a membership function. In addition, each Takaki-Sugeno type rule requires three parameters, which depend on the number of input variables, and all of these parameters need to be estimated using training data. We have used an artificial neural network (ANN) method for training our FIS. We refer the reader to Jang (1993) for further details.

In order to validate and verify the above model for our case study we chose the historically early part of the available data to be the training data and use it to validate the results. Subsequently, the unused later part of the data is used to verify the performance of the model. In the next section we discuss the chosen case study and present results corresponding to both the regression method and the FIS.

\subsection{Results and Discussion}

A single reservoir example is shown in Figure 9.3. A reservoir is used to augment flow in a creek such that the water quality, for example, dissolved oxygen (DO) is maintained above a certain required value (for example, $5 \mathrm{mg}$ / 1). From previous data analysis, optimization (Fletcher and Ponnambalam, 1998) and the simulation of optimal releases we have found target releases such that this objective is achieved. The objective of FIS is to use these optimal releases, corresponding storage volumes, and inflows to come up with system parameters such that the use of FIS is expected to produce similar benefits in the future. Depending upon the length of the training data the FIS or, for that matter, the regression analysis is expected to perform differently. In general, 


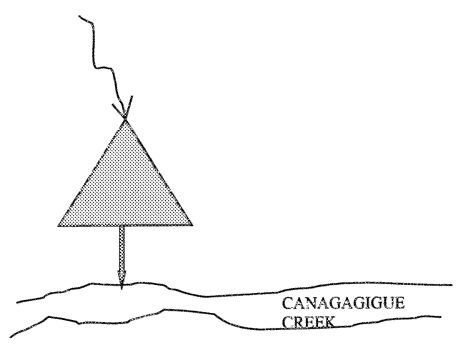

Figure 9.3 Flow augmentation to reduce pollutant concentration in a creek through controlled release.

the longer the training data, the better the performance as shown in Figure 9.4. The error here is the sum of squared deviations of real releases from target releases using the corresponding decision making system. It is clear that, in terms of relative error, the FIS has far better performance than the regression analysis (about $400 \%$ better).

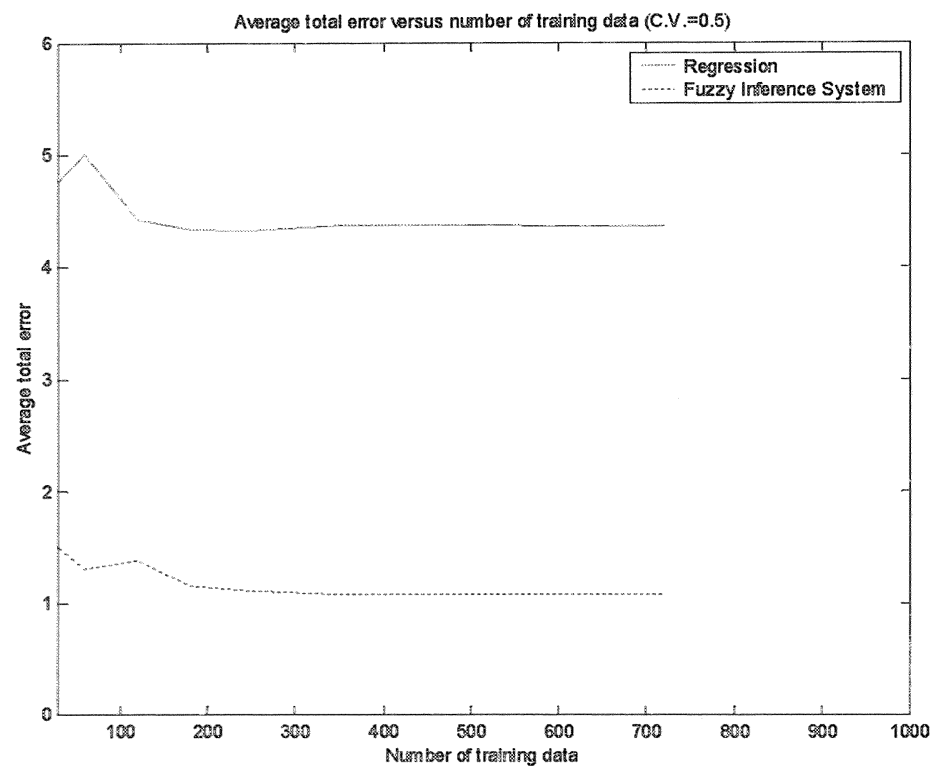

Figure 9.4 Least-square error from the application of release policies.

If the problem is linear, it is expected that the higher the uncertainty (that is, the higher the coefficient of variation) the worse the performance will become. However, the results in Figure 9.5 do not support this simplistic assumption, probably due to the nonlinearity in the problem. Although the FIS 


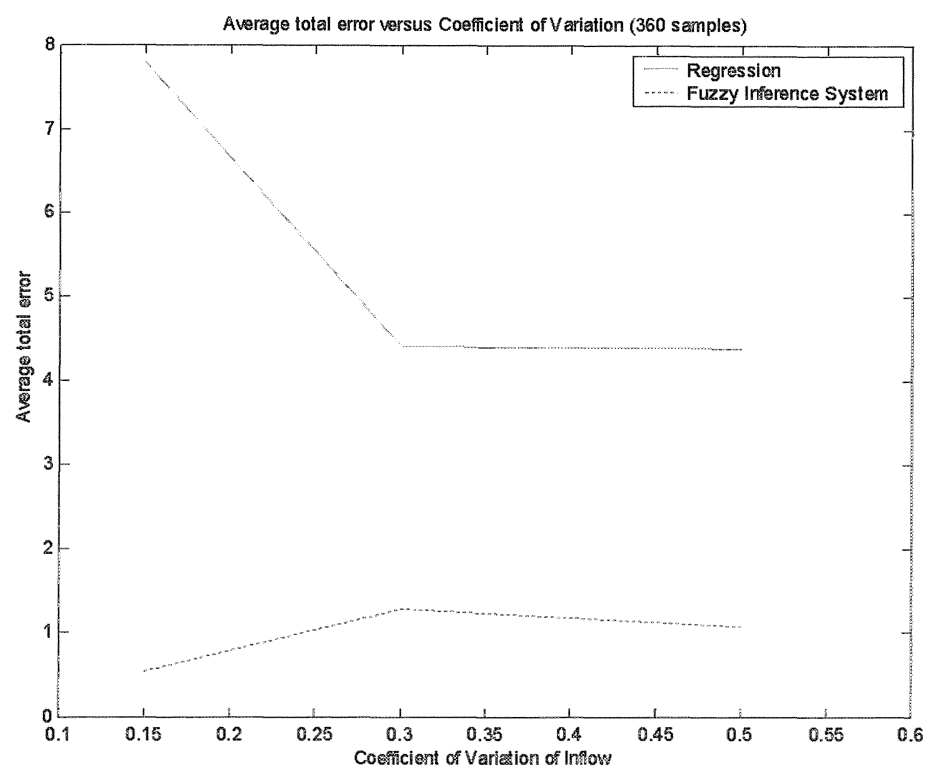

Figure 9.5 Variation of error with respect to changes in the coefficient of variation in inflows.

performed better, it should be noted that the regression method has seven parameters per season, the FIS needed to fit about 21 parameters for each season. Moreover, the fitting method for regression is much simpler than that used in the FIS which is based on artificial neural networks.

\subsection{Conclusion}

A Fuzzy Inference System was developed for reservoir operation based on optimal releases to maintain water quality in a creek. A regression-based method was also developed for the same purpose. Possibly due to nonlinearity in reservoir system equation, the FIS performs much better than the regression method. Fuzzy inference systems can also use the knowledge of experts through either directly using past operations or using survey methods and hence may be better suited for real-world operations. The caveat is that FIS requires a larger number of parameters and a more sophisticated fitting method for parameter estimation than does the regression method. 


\section{References}

Fletcher, S. G., and K. Ponnambalam (1998) "A constrained state formulation for the stochastic control of multireservoir systems.” Water Resources Research, 34(2), pp. 257-269

Jang, J.-S. R. (1993) “ANFIS: Adaptive -Network-based Fuzzy Inference Systems.” IEEE Transactions on Systems, Man, and Cybernetics, 23(3), pp. 665-685.

Sugeno, M. (1985) "Industrial applications of fuzzy control." Elsevier Science Pub. Co., Amsterdam.

Zimmermann, H. -J. (1984) "Fussy sets and decision analysis." Elsevier Science, NewYork,Amsterdam. 